\title{
Image Improvement from a Sodium-Layer Laser Guide Star Adaptive Optics System
}

C.E. Max, S.S. Olivier, H.W. Friedman, J. An, K. Avicola, B.V. Beeman, H.D. Bissinger, J.M. Brase, G.V. Erbert, D.T. Gavel, K. Kanz, M.C. Liu, B. Macintosh, K.P. Neeb, J. Patience and K.E. Waltjen

June 1997

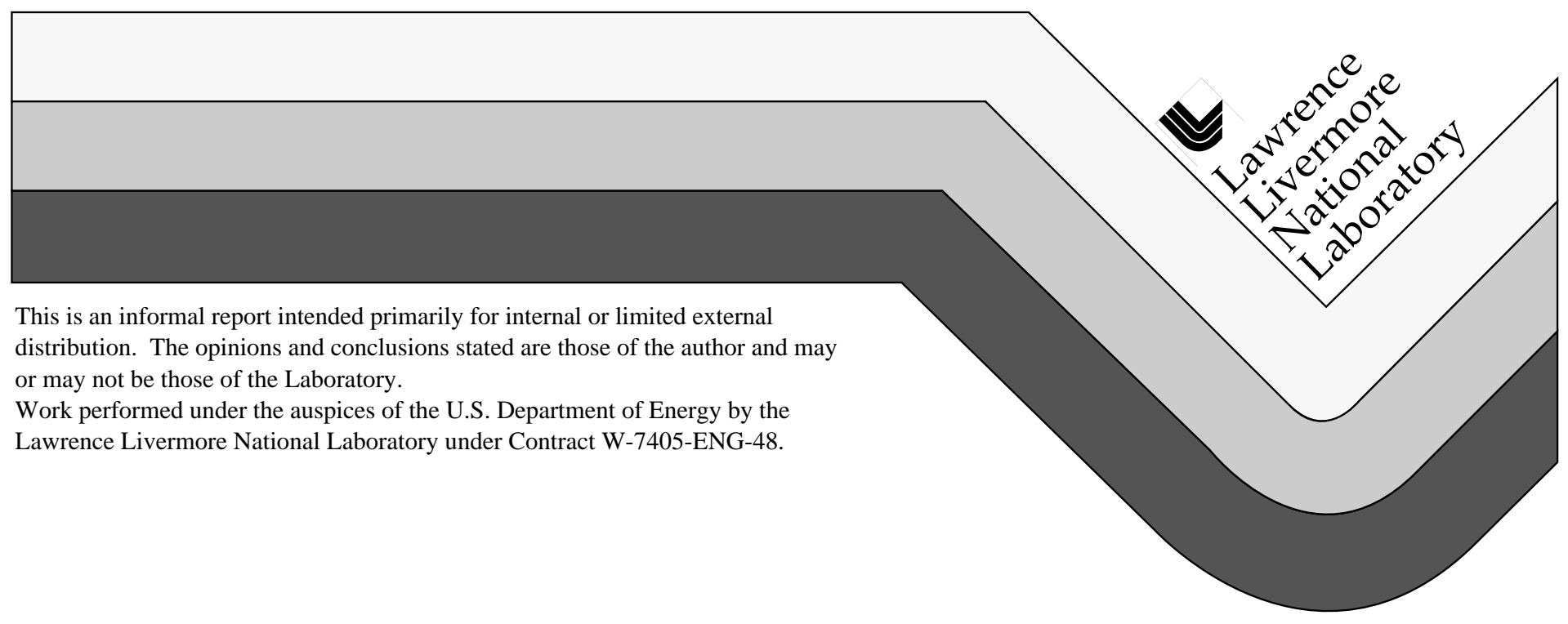




\section{DISCLAIMER}

This document was prepared as an account of work sponsored by an agency of the United States Government. Neither the United States Government nor the University of California nor any of their employees, makes any warranty, express or implied, or assumes any legal liability or responsibility for the accuracy, completeness, or usefulness of any information, apparatus, product, or process disclosed, or represents that its use would not infringe privately owned rights. Reference herein to any specific commercial product, process, or service by trade name, trademark, manufacturer, or otherwise, does not necessarily constitute or imply its endorsement, recommendation, or favoring by the United States Government or the University of California. The views and opinions of authors expressed herein do not necessarily state or reflect those of the United States Government or the University of California, and shall not be used for advertising or product endorsement purposes.

This report has been reproduced directly from the best available copy.

Available to DOE and DOE contractors from the Office of Scientific and Technical Information P.O. Box 62, Oak Ridge, TN 37831

Prices available from (423) 576-8401

Available to the public from the National Technical Information Service

U.S. Department of Commerce 5285 Port Royal Rd. Springfield, VA 22161 


\title{
Image improvement from a sodium-layer laser guide star adaptive optics system
}

\author{
C. E. Max, S. S. Olivier, H. W. Friedman, J. An, K. Avicola, \\ B. V. Beeman, H. D. Bissinger, J. M. Brase, G. V. Erbert, D. T. Gavel, \\ K. Kanz, M. C. Liu, B. Macintosh, K. P. Neeb, J. Patience, and K. E. Waltjen.
}

A sodium-layer laser guide star beacon with high-order adaptive optics at Lick Observatory produced a factor of 2.4 intensity increase and a factor of 2 decrease in full width at half maximum for an astronomical point source, compared with image motion compensation alone. Image full widths at half maximum were identical for laser and natural guide stars ( 0.3 arc seconds). The Strehl ratio with the laser guide star was $65 \%$ of that with a natural guide star. This technique should allow ground-based telescopes to attain the diffraction limit, by correcting for atmospheric distortions.

C. E. Max, S. S. Olivier, H. W. Friedman, J. An, K. Avicola, B. V. Beeman, H. D. Bissinger, J. M. Brase,

G. V. Erbert, D. T. Gavel, K. Kanz, B. Macintosh, K. P. Neeb, and K. E. Waltjen, Lawrence Livermore National Laboratory, 7000 East Avenue, Livermore, California, 94550

M. C. Liu, Department of Astronomy, University California at Berkeley, Berkeley, California, 94720

J. Patience, Department of Physics \& Astronomy, University California at Los Angeles, 405 Hilgard Avenue, Los Angeles, California 90095 
Images of astronomical objects are distorted by variations in the index of refraction of air due to atmospheric turbulence. These aberrations limit the resolution (for example the full width at half maximum, FWHM) of long-exposure images to $\sim 0.5$ arc sec at visible and near-infrared wavelengths. If the effects of turbulence could be fully corrected, telescopes of diameter 1 to $10 \mathrm{~m}$ would achieve resolutions of 0.1 to 0.01 arc sec at $0.5 \mu \mathrm{m}$ wavelength. Adaptive optics systems which sense and correct atmospheric aberrations were proposed by Babcock in 1953 (1). About 10 such systems are now installed on astronomical telescopes (2), including one developed for the $3 \mathrm{~m}$ Shane Telescope at Lick Observatory (3). These systems use a natural star as reference beacon. However, requirements on reference beacon brightness and proximity typically restrict the use of natural guide star adaptive optics systems to a small fraction $(<10 \%)$ of the sky.

In principle, laser guide star systems can create reference beacons anywhere on the sky. Two techniques to generate the laser beacon are being pursued. The first uses Rayleigh scattering from air molecules at altitudes of $10-15 \mathrm{~km}$. A system using this technique is in operation on a $1.5 \mathrm{~m}$ telescope at the Starfire Optical Range (4). Because Rayleigh scattering is limited to $<15 \mathrm{~km}$ height by the exponential decrease in air density, the wavefront from a Rayleigh beacon does not sample the same aberrations as the wavefront from an astronomical object. This is called the cone effect because the Rayleigh beacon samples a cone instead of a cylinder. The cone effect, also known as focus anisoplanatism (5), is more pronounced for a loweraltitude reference beacon and for a larger telescope. For telescopes with diameters of 6 to $10 \mathrm{~m}$, the cone effect from a Rayleigh beacon limits the ability to correct for atmospheric turbulence. 
The second technique is the use of laser-produced resonance fluorescence of atomic $\mathrm{Na}$ in the mesosphere at a height of $\sim 95 \mathrm{~km}$ (laser wavelength $589 \mathrm{~nm}$ corresponding to the $\mathrm{Na}_{2}$ line) $(6,7)$. The $95 \mathrm{~km}$ height of the Na layer reduces the cone effect, compared to a Rayleigh beacon. Passive high-order (8) and low-order (9) wavefront phase measurements using a sodium-layer laser guide star have been performed. Active image improvement using a sodium-layer laser guide star with a low-order image-stacking system on the Multiple Mirror Telescope was accomplished in 1994 (10). Here we report image improvement with a high-order adaptive optics system and a sodium-layer laser guide star.

The Lick Observatory adaptive optics system (3) is mounted at the f/17 Cassegrain focus (f-number is a measure of image brightness) of the $3 \mathrm{~m}$ Shane Telescope (11). The adaptive optics system (Fig. 1) feeds LIRC-2, a near-infrared camera based on a HgCdTe 256x256 pixel detector.

The deformable mirror corrects wavefront phase over the telescope pupil. This thin glass mirror has 127 electro-restrictive (lead magnesium niobate) actuators in a triangular pattern, each capable of deforming the front surface by up to $\pm 4 \mu \mathrm{m} ; 61$ of the actuators are actively controlled. A separate flat fast-steering mirror is used to correct the overall wavefront tip-tilt (image motion).

The Shack-Hartmann wavefront sensor (12) has 37 subapertures in the clear aperture of the telescope (subaperture diameter $44 \mathrm{~cm}$ mapped to the primary mirror). The wavefront sensor uses a $64 \times 64$ pixel CCD, with read noise of $7 \mathrm{e}^{-}$per pixel at 1200 frames s$^{-1}$. In laser guide star mode a separate sensor is used for tip-tilt (13), with 4 photon-counting avalanche photo-diodes configured as a quad cell. A 
dedicated analog controller uses input from this tip-tilt sensor to control the faststeering mirror in the adaptive optics system, at a bandwidth up to $120 \mathrm{~Hz}$.

The wavefront control computer is a 160 Mflop Mercury system with 4 Intel i860 processors. It is operated at a sample rate up to $500 \mathrm{~Hz}$ with control bandwidth $(0 \mathrm{~dB}$ crossover) up to $30 \mathrm{~Hz}$, including camera integration and readout rate, compute rate, and transfer rate to the deformable mirror drivers. In laser guide star mode, the average wavefront sensor tip-tilt is utilized by a separate digital processor to control a high-bandwidth steering mirror within the laser launch optics, stabilizing the laser guide star on the wavefront sensor.

The laser guide star (14) is a tunable dye laser pumped by flash-lamp-pumped frequency-doubled Nd:YAG lasers. Pump lasers are located in a room below the telescope dome and fiber-optically coupled to the dye laser on the side of the $3 \mathrm{~m}$ telescope (Fig. 2). The dye laser produces light tuned to $589 \mathrm{~nm}$ which is projected into the sky by a refractive launch telescope with $30 \mathrm{~cm}$ primary lens (15). The laser projects $18 \mathrm{~W}$ of average power with pulse width of $\sim 100 \mathrm{~ns}$ and pulse repetition rate of $\sim 11 \mathrm{kHz}$.

In our experiments of Sept. - Oct. 1996 the measured value of the atmospheric coherence length $\mathrm{r}_{0}$ was $\sim 11 \mathrm{~cm}$ at $0.55 \mu \mathrm{m}$. The apparent size of the guide star in the Na layer was measured in $10 \mathrm{sec}$ exposures to be $\sim 2$ arc sec. The return signal from the laser guide star was comparable to that for a natural star with magnitude 7.0 in $\mathrm{V}$ band $(\lambda=5550 \AA, \Delta \lambda \sim 1000 \AA)$. This return signal is a factor of 2 higher than measured at LLNL in 1992 (8) for comparable laser power, and a factor of $\sim 4$ higher than previous measurements at Lick. Factors of $2-4$ changes in return flux are expected due to seasonal and night-to-night variation in sodium column density. 
The field stars $\gamma$ Trianguli and SAO 56102 were observed with the infrared camera at exposure times of $20 \mathrm{~s}$ and $60 \mathrm{~s}$ respectively (Fig. 3). Compared with tip-tilt correction alone, laser guide star adaptive optics increased the peak intensity by a factor of 2.4 and decreased its FWHM by a factor of 2. Relative to the uncorrected images, peak intensity was increased by a factor of 1.4 for tip-tilt correction alone (16), by 4.2 for tip-tilt with natural guide star adaptive optics, and by 3.3 for tip-tilt with laser guide star adaptive optics. The FWHM was decreased by a factor of 1.3 (from 0.8 to 0.6 arc sec) for tip-tilt correction alone, and by a factor of 2.7 (to 0.3 arc sec) for natural and laser guide star correction. Both laser guide star and field star were onaxis. Light from the field star was attenuated on the wavefront sensor using a narrow-band filter centered at $589 \mathrm{~nm}$. The field star was used as the tip-tilt guide star. Images (Fig. 3) were made at about $5^{\circ}$ from zenith with sampling frequency of $200 \mathrm{~Hz}$. Similar image improvement was obtained on 30 Sept. and 1 Oct. as well as on 26 and 27 Nov., 1996, the latter under seeing conditions worse by a factor of 2-3 with 30-40 mph surface winds. All nights showed little temperature variation; there was no significant contribution due to correction of primary mirror aberrations.

The encouraging nature of our results is illustrated by the fact that the increase in peak intensity with laser guide star adaptive optics is $75 \%$ of the increase for natural guide stars, with all other system parameters (including tip-tilt) held constant. The corrected FWHM was identical for the laser and natural guide stars. In other words, high-order correction using the laser guide star was nearly as good as that using a natural star.

The power spectrum of wavefront phase fluctuations measures the power per unit frequency interval present in residual uncorrected phase aberrations. The open and 
closed-loop phase power spectra (Fig. 4) for a natural guide star and a laser guide star are similar. For both systems adaptive optics decreases residual phase fluctuations at low frequencies, with the correction losing its impact at frequencies greater than 5-10 Hz. The noise floor above $10 \mathrm{~Hz}$ (Fig. 4) is due to the fact that above a certain frequency, the noise is larger than the signal. The noise floor for the laser guide star is higher than for the natural guide star, because the laser spot is larger and dimmer.

Strehl is a measure of image quality: the ratio of measured peak intensity to that of a theoretical image limited only by diffraction. The Strehl of the laser guide star corrected image is $0.091,65 \%$ of that measured for the natural guide star (Table 1 ). Ten percent of this difference is due to poorer performance of the tip-tilt system (discussed further below). The remainder is due to differences in size and shape on the wavefront sensor between the laser guide star and the natural star, and to focus error introduced when the height of the sodium layer is not perfectly sensed.

The Strehl ratio can be decomposed into six contributing sources of residual aberration (17) (Table 1). Fitting error is due to limitations on the ability of the deformable mirror to respond to high-spatial-frequency variations of the atmosphere, because of the finite number of actuators. The fitting error was estimated using a standard scaling law from atmospheric turbulence theory (18) with atmospheric coherence length from the uncorrected image FWHM. Servo error is due to the finite bandwidth of the control loop, and was calculated from the integral of the measured phase power spectrum after removal of the noise floor. Measurement error is due to the finite ability of the wavefront sensor to measure the wavefront in the presence of noise. Measurement error was calculated from the integral of the power spectrum noise floor, taking into account filtering of the noise by the control loop (19). Because the total measurement error is quite small for 
these experiments, finite laser guide star spot size is not a significant factor (20). Tiptilt error (servo- plus measurement-error) represents the finite ability of the tip-tilt system to stabilize image motion. For the natural guide star, tip-tilt error is calculated from power spectra of wavefront sensor centroid positions averaged over the pupil, after taking into account the intrinsic measurement error of the average centroid position. For the laser guide star the average centroid position is affected by the deviation of the laser beam as it goes upwards. Tip-tilt measurement error for the laser was calculated by assuming the same servo error as for the natural guide star, and scaling the measurement error by the measured decrease in the sum voltage signal on the tip-tilt sensor quad cell. Calibration error is due to residual non-common-path aberration between the wavefront sensor and science camera focal planes. Cone effect measures the difference between the wavefront measured from the finite-altitude laser guide star and from a real star located an "infinite" distance away. Where appropriate, the Strehl ratios (Table 1) are approximated by $\exp \left(-\sigma^{2}\right)$ where $\sigma$ is the root-mean-square wavefront error in radians, at $2.2 \mu \mathrm{m}$.

Residual error in calibration of non-common path optics arises from the adaptive optics system, and from inside the IR camera dewar. Calibration error for the adaptive optics system was measured using a white-light reference source and a CCD camera with a narrow-band filter centered at $700 \mathrm{~nm}$. Calibration error for the IR camera was measured using the white-light source and two different filters within the IR camera. The filter with narrower bandwidth, used for the brighter natural guide star, had slightly more measured error. Calibration error was minimized by adjusting the deformable mirror to produce the best reference image.

For laser and natural guide stars, the measured Strehl was about 0.6 that predicted from the measured error sources. This implies that there was a secular drift in 
calibration over the 6 hour interval between the last calibration and image data collection. The drift is due to flexure from temperature effects and other mechanical stresses, as well as inaccuracies in repositioning the wavefront sensor between the focal positions for the natural guide star and for the laser star. These errors can be reduced by calibrating more frequently.

With improved calibration, the sodium guide star technique is predicted to perform very well even for the largest telescopes: with a sodium-layer laser guide star system, the 10-m Keck Telescopes should have limiting tip-tilt magnitude $\mathrm{m}_{\mathrm{V}}=19$ with angular resolution of 0.02 arc sec in the near infra-red (21). 


\section{NOTES AND REFERENCES}

1. H. W. Babcock, Publ. Astron. Soc. Pacific 65, 229 (1953); ibid. Science 249, 253 (1990).

2. L. A. Thompson, Physics Today 47, 24 (1994).

3. J. M. Brase, J. An, K. Avicola, H. D. Bissinger, H. W. Friedman, D. T. Gavel et al., Proc. SPIE 2201, 474

(1994); S. S. Olivier, J. An, K. Avicola, H. D. Bissinger, J. M. Brase, H. W. Friedman et al., Proc. SPIE 2201, 1110 (1994).

4. R. Q. Fugate, D. L. Fried, G. A. Ameer, B. R. Boeke, S. L. Browne, P. H. Roberts et al., Nature 353144 (1991).

5. D. Fried, J. Opt. Soc. Am. A 11, 277 (1994); D. Fried and J. F. Belsher, J. Opt. Soc. Am. A 11, 227 (1994).

6. W. Happer, G. MacDonald, C. E. Max, F. Dyson, J. Opt. Soc. Am. A 11, 263 (1994).

7. R. Foy and A. Labeyrie, Astron. Astrophys. 152, L29 (1985).

8. K. Avicola, J. M. Brase, J. R. Morris, H. D. Bissinger, J. M. Duff, H. W. Friedman et al., J. Opt. Soc. Am. A11, 825 (1994).

9. R. A. Humphreys, C. A. Primmerman, L. C. Bradley, J. Herrmann, Opt. Lett. 16, 1367 (1991). Measurements of laser guide star centroid motion were made in 2 contiguous subapertures.

10. M. Lloyd-Hart, J. R. P. Angel, B. Jacobsen, D. Wittman, R. Dekany, D. McCarthy et al., Astrophys. J. 439, 455 (1995). This system allowed control of relative tip-tilt between 2 (and subsequently, all 6) of the Multiple Mirror Telescope's $1.8 \mathrm{~m}$ primary mirrors.

11. S. S. Olivier, J. An, K. Avicola, H. D. Bissinger, J. M. Brase, H. W. Friedman et al., Proc. SPIE 2534, 26 (1995).

12. A Shack-Hartmann sensor divides the telescope pupil into subapertures using a set of miniature lenses (lenslet array) placed in a reimaged pupil plane. The average wavefront slope in each subaperture is determined by measuring the position of the focused image formed by each lenslet. The constraint of continuity can then be imposed, and an estimate of the wavefront phase can be reconstructed, for example, from a least-squares fit.

13. Because the laser travels up through the turbulent atmosphere before forming the guide star, the instantaneous physical position of the guide star is variable. This upgoing beam wander results in a difference between the apparent instantaneous positions of a laser guide star and a natural star, rendering the laser guide star unsuitable as a reference for stabilization of the overall image position of astronomical objects. The solution is to 
use a faint natural star as overall tip-tilt reference. If this technique is employed, the sky coverage for a laser guide star adaptive optics system is limited by the availability of suitable tip-tilt reference stars [S. S. Olivier, C. E. Max, D. Gavel, J. Brase, Astrophys. J. 407, 428 (1993); S. S. Olivier and D. T. Gavel, J. Opt. Soc. Am. A 11, 368 (1994)]. Because the requirements for a tip-tilt reference star are less severe than for a high-order wavefront reference beacon, use of a laser guide star increases the sky coverage fraction for adaptive optics systems.

14. H. Friedman, G. Erbert, T. Kuklo, T. Salmon, D. Smauley, G. Thompson, N. Wong, Proc. SPIE 2201, 352 (1994); H. W. Friedman, G. V. Erbert, T. C. Kuklo, J. G. Malik, J. T. Salmon, D. A. Smauley et al., Proc. SPIE 2534, 150 (1995).

15. H. W. Friedman, G. V. Erbert, D. T. Gavel, T. C. Kuklo, J. G. Malik, J. T. Salmon et al., Proc. European Southern Observatory, Conference No. 54, (ESO, Garching, Germany, 1995), p. 207.

16. For the measured seeing conditions $\left(0.8 \operatorname{arc~sec}\right.$ at $\lambda=2.2 \mu \mathrm{m}, \mathrm{r}_{0}=10.7 \mathrm{~cm}$ at $\left.\lambda=0.55 \mu \mathrm{m}\right)$, direct integration of the modulation transfer function for telescope and ensemble-averaged atmosphere [ D. L. Fried, J. Opt. Soc. Am. 56, 1372 (1966), D. T. Gavel and S. S. Olivier, Proc. SPIE 2201, 295 (1994)] shows that the expected increase in peak intensity for tip-tilt correction is a factor of 3.7. However, taking into account the measured and inferred calibration errors of $1.7 \mathrm{rad}^{2}$ (assuming they have roughly the same spectrum as the tiltcorrected atmosphere) the expected intensity increase is only a factor of 1.7. The measured value was 1.4. The degraded tip-tilt performance is attributable to misalignment of the tip-tilt sensor and to non-optimal tuning of the tip-tilt control parameters.

17. For a review see J. M. Beckers, Ann. Rev. Astron. Astrophys. 31, 13 (1993).

18. D. L. Fried, J. Opt. Soc. Am. 55, 1427 (1965).

19. The factor by which the rms noise is reduced due to averaging by the control loop was estimated to be $\chi=$ $\left[(2 / \kappa) \tan ^{-1}(\kappa / 2)\right]^{1 / 2}$, where $\kappa$ is the ratio of the sampling frequency to the control bandwidth [S. S. Olivier, C. E. Max, D. Gavel, J. Brase, Astrophys. J. 407, 428 (1993)].

20. Apart from the impact of finite laser spot size on measurement error (negligible in our experiments because of the very high signal to noise ratio), anisoplanatism due to finite laser spot size (R. Sasiela, Lincoln Laboratory Technical Report no. 807, 1988) contributes a mean square error of $\sigma^{2}=\left(0.1014 \theta_{\mathrm{gs}} / \theta_{0}\right)^{5 / 3}<0.01$ for the parameters of our experiment, where $\theta_{\mathrm{gs}} \sim 2$ arc sec is the laser spot size in the mesosphere and $\theta_{0}=3-5$ arc sec is the isoplanatic angle. Hence finite laser spot size is not a significant factor in our data. 
21. C. E. Max, D. T. Gavel, S. S. Olivier, J. Brase, in Adaptive Optics for the Keck Observatory (W. M. Keck Observatory, Waimea, HI 1995), report. no. 208, chapter 4.

This work was performed under the auspices of the U.S. Department of Energy by the Lawrence Livermore National Laboratory under Contract number W 7405-Eng-48. 


\section{FIGURE CAPTIONS}

Fig. 1. Lick adaptive optics system schematic. (A) Front view. Light from the telescope enters from top center on the diagram and hits two turning mirrors, the tip-tilt mirror, an off-axis parabolic collimating mirror, and the deformable mirror. The light then hits a dichroic beam splitter that reflects wavelengths shorter than $600 \mathrm{~nm}$ to the wavefront sensor leg consisting of an off-axis parabolic focusing mirror, a turning mirror, a collimating lens, the lenslet array, a set of relay telescope lenses, and finally the wavefront sensor camera. Light at wavelengths longer than $600 \mathrm{~nm}$ passes through the dichroic beam splitter to the science camera leg, consisting of an off-axis parabolic focusing mirror, a turning mirror that sends the light to the back side of the optical bench. (B) Rear view. A turning mirror receives the light from the front side of the optical bench, and a dichroic beam splitter transmits wavelengths longer than $900 \mathrm{~nm}$ to a turning mirror and thence to an infrared science camera, LIRC-2. Wavelengths shorter than $900 \mathrm{~nm}$ are reflected from the dichroic beam splitter to a turning mirror. A variable beam splitter after the turning mirror passes a selectable fraction of the light to the tip-tilt sensor and reflects the rest to a beam splitter cube. Half the light from this cube goes to a CCD scoring camera, and the other half to a low-speed wavefront sensor. The latter is used to monitor focus and other slowly varying aberrations introduced by the laser guide star.

Fig. 2. LLNL laser guide star installation at Lick Observatory. The dye master oscillator and the Nd:YAG pump lasers are in a room below the dome floor. An umbilical tube from this room to the telescope contains the lines that carry the dye, as well as optical fibers carrying high-power green YAG light and low-power yellow light from the dye master oscillator to the dye laser amplifier on the telescope. High-power yellow light from the dye laser amplifier travels up through the diagnostics package that monitors beam pointing and wavefront quality, and propagates into the sky through the launch telescope.

Fig. 3. Image data without (left) and with (right) correction using (A) the tip-tilt system alone, (B) both the tip-tilt and high-order adaptive optics system with a natural guide star, and (C) both the tip-tilt and high-order adaptive optics system with the sodium-layer laser guide star. These images of the field stars (A), (B) $\gamma$ Trianguli (20 s exposures) and (C) SAO 56102 (60 s exposures) were recorded within an hour on October 1, 1996 at a 
wavelength of $2.2 \mu \mathrm{m}$. The image peak intensities after correction by adaptive optics are (A) 1.4, (B) 4.2, and (C) 3.3 times the respective uncorrected values. The corrected image widths are (A) 0.6 and (B), (C) 0.3 arc seconds, a factor of (A) 1.3 and (B), (C) 2.7 less than the uncorrected size of 0.8 arc seconds. The increase in peak intensity using the laser guide star is $75 \%$ of that using the natural guide star, with other system parameters (including tiptilt) held constant. (The uncorrected image for $\gamma$ Trianguli in (A, B) has a normalized peak intensity $18 \%$ higher than that for SAO 56102 in (C), due to minor differences in exposure times, calibration, and atmospheric conditions.)

Fig. 4. Reconstructed phase power spectra for (A) the bright natural star, $\gamma$ Trianguli, and (B) the sodium-layer laser guide star. In each panel the upper curve was calculated from data taken with the control loop open, the lower curve from data with the control loop closed (Oct. 1, 1996). Laser guide star and natural guide star data were recorded at approximately the same time as the images shown in Figure 3. Because the Hartmann sensor used 4x4 pixels ( 1.8 arc sec per pixel or 7.2 arc sec width of the quad cell overall) for centroid determination, the dynamic range of the wavefront sensor was more than enough to accommodate the open-loop measurements, which showed r.m.s. image motion of about 1 arc sec. Each power spectrum was calculated from a series of 4096 wavefront sensor centroid measurements at a sampling frequency of $200 \mathrm{~Hz}$. The control bandwidth ( $0 \mathrm{db}$ crossover) was $\sim 10 \mathrm{~Hz}$. This measures the highest speed at which the control loop can correct phase aberrations. Atmospheric coherence time $\tau_{0}$ was 250 - $500 \mathrm{msec}$. 
Table 1. Residual errors for adaptive optics correction using both a natural and laser guide star.

\begin{tabular}{|c|c|c|}
\hline & $\begin{array}{c}\text { Natural guide star } \\
\text { adaptive optics } \\
\text { Strehl ratio }\end{array}$ & $\begin{array}{c}\text { Laser guide star } \\
\text { adaptive optics } \\
\text { Strehl ratio } \\
\end{array}$ \\
\hline Measured Strehl from corrected image & 0.14 & 0.091 \\
\hline Measured Strehl from uncorrected image & 0.028 & 0.028 \\
\hline \multicolumn{3}{|l|}{ Predicted or measured Strehl from individual } \\
\hline \multicolumn{3}{|l|}{ wavefront error sources: } \\
\hline 1. Fitting error & 0.82 & 0.82 \\
\hline 2. Servo error & 0.81 & 0.89 \\
\hline 3. Measurement error & 0.98 & 0.95 \\
\hline 4. Tip-tilt error & 0.76 & 0.66 \\
\hline \multicolumn{3}{|l|}{ 5. Calibration errors: } \\
\hline 5.1. Internal to $A O$ system & 0.89 & 0.89 \\
\hline 5.2. Internal to IR camera & 0.52 & 0.57 \\
\hline 6. Predicted cone effect & - & 0.98 \\
\hline Total predicted Strehl after correction & 0.23 & 0.23 \\
\hline Inferred internal calibration drift & 0.6 & 0.6 \\
\hline Inferred laser guide star calib. error & - & 0.65 \\
\hline
\end{tabular}




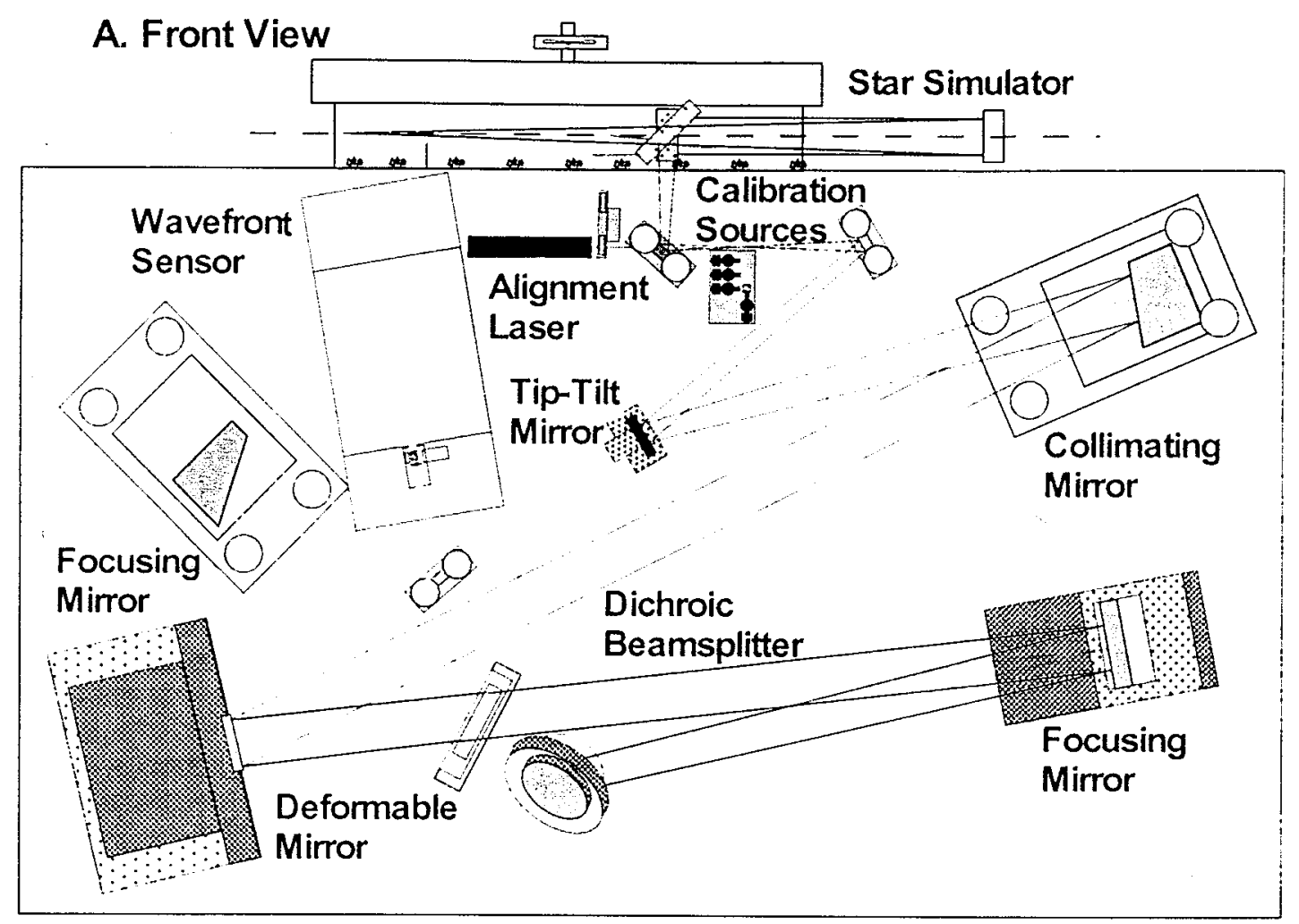

B. Back View

Tip-Tilt Sensor \& Alignment Camera

Variable Scoring Wavefront Sensor
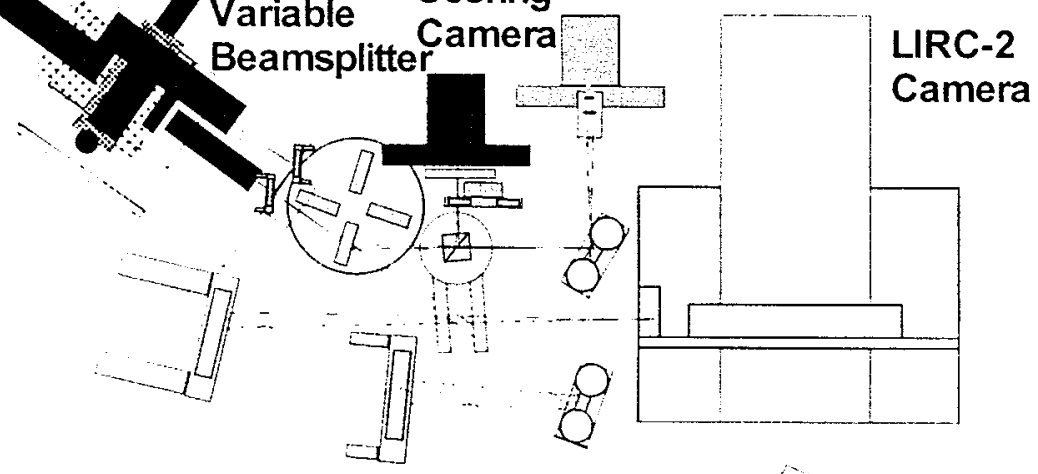

Dichroic

Beamsplitter

\section{Camera}




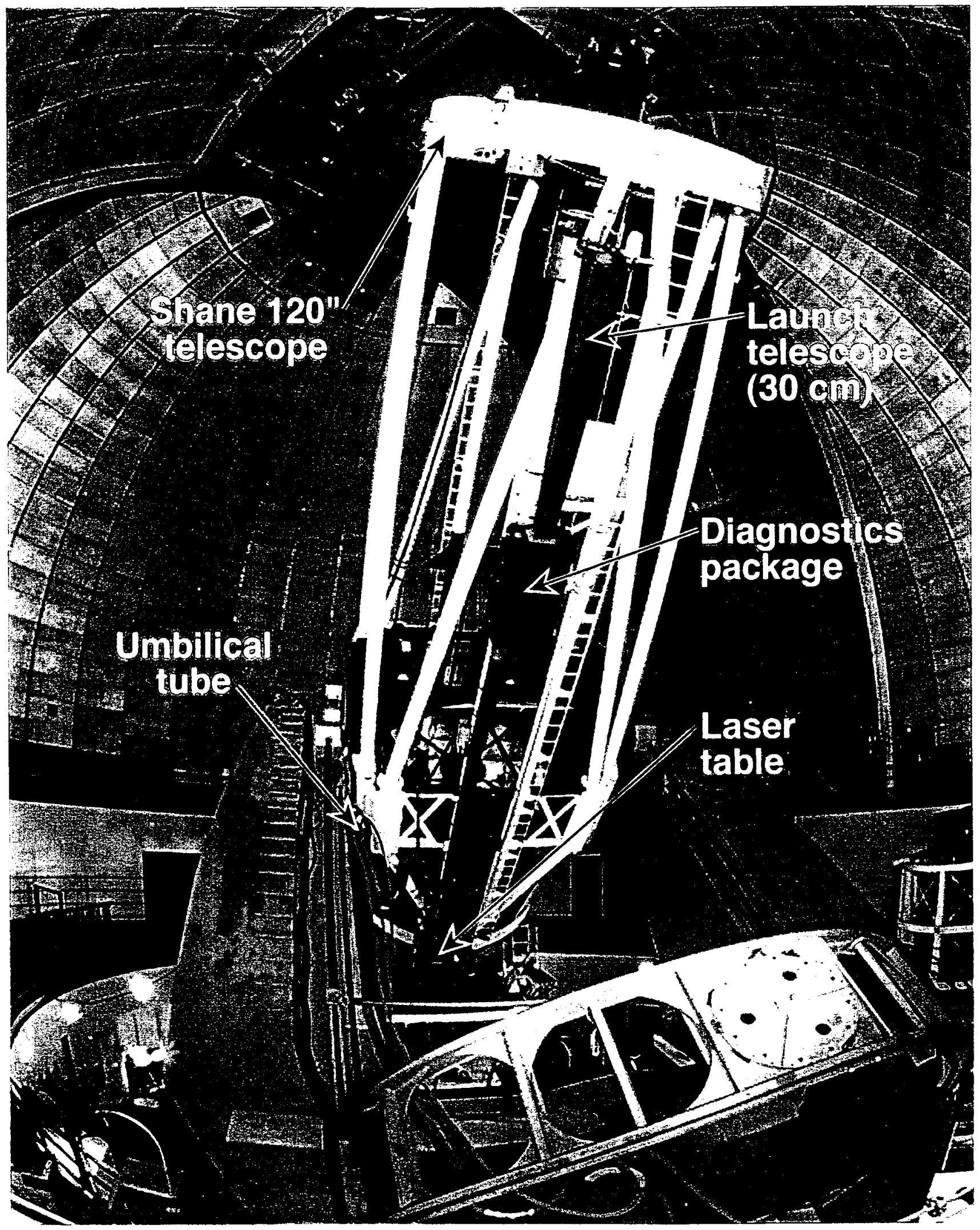





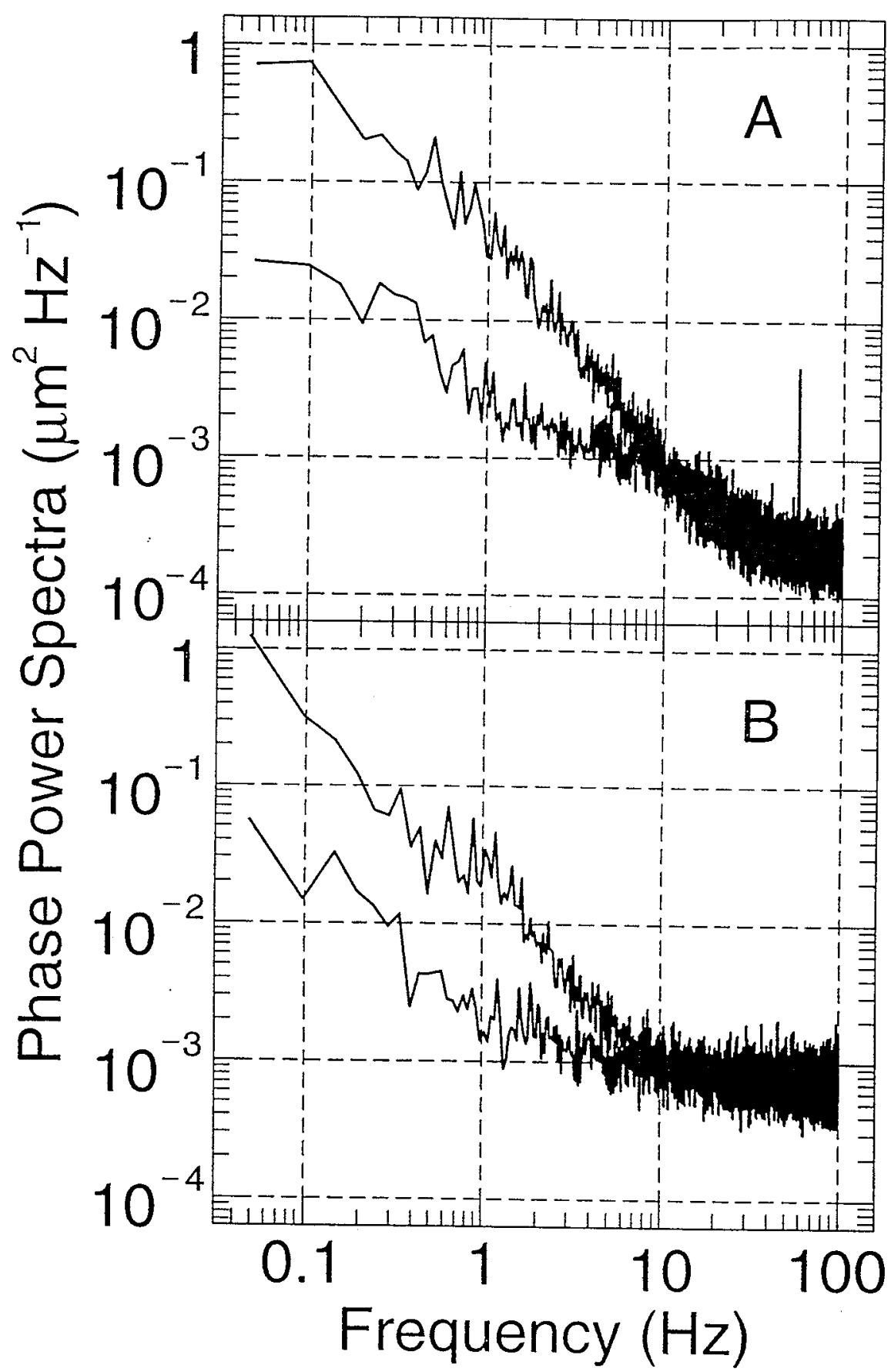




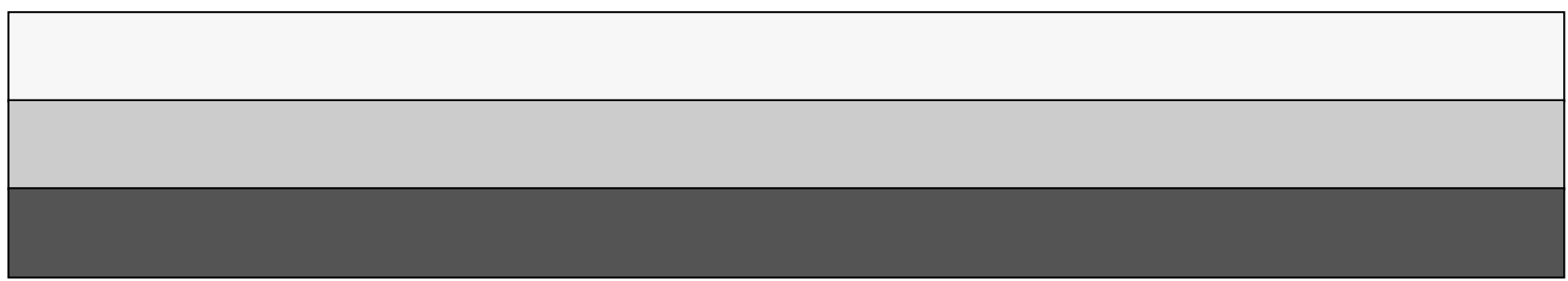

